\title{
A method of symmetrization of asymmetric dynamical systems
}

\author{
C.Q. Liu \\ DaimlerChrysler Corporation, CIMS 481-47-10, 800 Chrysler Drive East, Auburn Hills, MI 48326-2757, USA \\ E-mail: cl31@dcx.com
}

Received 30 June 2004

Accepted 10 November 2004

\begin{abstract}
This paper presents an approach to transform asymmetric systems into symmetric systems by equivalence transformation and discusses what forms of restrictions should be imposed on the system matrices so that they can be simultaneously transformed into symmetric matrices. Conditions of symmetrizability obtained here are more "liberal" and numerical calculations of this transformation are more straightforward. Several examples are provided to illustrate the new approach.
\end{abstract}

\section{Introduction}

Theory of linear symmetric systems has been well developed. However, for a general nonconservative system, the symmetry restrictions are not met by the coefficient matrices of governing equations. These matrices are merely square arrays of real numbers. Problems of this type arise in gyroscopic and circulatory systems [1], aircraft flutter [2], ship motion in sea water [3], actively controlled systems [4], as well as in constrained multi-body systems [5]. Many authors have considered these so-called "nonclassical systems" [6-12,14,15]. Recently, Adhikari [14] proposed a method to obtain (complex) eigensolutions of general asymmetric nonconservative systems without converting the equations of motion into first-order form. Without doubt, it would be preferable if asymmetric systems can be transformed into equivalent symmetric systems so that one can take advantage of the well-developed theories for symmetric systems to analyze them. It is therefore of interest to study "symmetrizability" of asymmetric systems. By Adhikari's definition [12], the method of symmetrization for asymmetric systems can be classified into two categories. That is, symmetrization of the first kind and of the second kind. The former is based on Taussky's factorization approach by similarity transformation. The latter is based on equivalence transformation.

In this paper we present an alternative method of symmetrization for asymmetric systems. This constructive method utilizes equivalence transformation, and avoids the calculations of Taussky's factorization as in symmetrization of the first kind and also avoids the calculations of eigenvalues and eigenvectors as in symmetrization of the second kind provided by Adhikari [12]. As will be evident, numerical calculations of the new method become more straightforward. The examples proposed by Adhikari [12] are considered and used to verify the presented results.

\section{Notations and definitions}

We use $R^{N \times N}$ to represent the space of $N \times N$ real matrices and $C^{N \times N}$ to represent the space of $N \times N$ complex matrices. A unit matrix $I \in R^{N \times N}$ is a diagonal matrix with all diagonal entries equal to one. Let $A \in C^{N \times N}$, then we use $A^{T}, \bar{A}, A^{-1}, A^{-T}$ and $A^{H}$ to represent the transpose, complex conjugate, inverse, inverse transpose, and transpose conjugate, respectively. A matrix is called symmetric if $A=A^{T}$, Hermitian if $A=A^{H}$, and unitary if $A A^{H}=I$. If $A$ is real, then a Hermitian matrix is equivalent to a symmetric matrix and a unitary matrix is equivalent to a real orthogonal matrix. Two matrices $A$ and $B$, related by $B=L^{T} A R$ for some nonzero $L$ and $R$, 
are called an equivalence transformation. When $L^{T}=R^{-1}$, the equivalence transformation is called a similarity transformation, and $A$ and $B$ are said to be similar. In the event $L=R$ then equivalence transformation is a congruence transformation. Classical modal transformation in symmetric systems is an example of congruence transformation.

\section{Symmetrizability of an asymmetric matrix}

By Taussky [13], symmetrizability of a matrix is defined as the following:

Definition 1: A matrix $A$ is symmetrizable if and only if any one of the following hold:

1. $A$ is the product of two symmetric matrices, one of which is positive definite;

2. $A$ is similar to a symmetric matrix;

3. $A^{T}=S^{-1} A S$ with $S=S^{T}>0$; and

4. A has real characteristic roots and a full set of characteristic vectors.

Adhikari [12] generalized Taussky's result and introduced the concept of symmetrizability of the second kind as following:

Definition 2: A matrix $A$ is symmetrizable of the second kind if and only if there exist two nonzero matrices $L$ and $R$ such that $\tilde{A}=L^{T} A R$ is a symmetric matrix.

This definition of symmetrizability is quite general and valid for both real and complex matrices. It also holds Taussky's definition as a special case when $L^{T}=R^{-1}=R^{-T}$.

One of the main results in Adhikari [12] is the following theorem:

Theorem 1: ([12] Theorem 4.1) Every rectangular complex matrix $A \in C^{r \times s}$ can be transformed to a real symmetric (square) matrix by an equivalence transformation.

In the following, we will present an alternative proof of this theorem. As will be seen, this proof itself provides a straightforward procedure for symmetrization of an asymmetric matrix. In fact, performing singular value decomposition, we have

$$
A=U S V^{T}
$$

where $U \in C^{r \times r}$ and $V \in C^{s \times s}$ are unitary matrices, and where $S \in R^{r \times s}$ is a real matrix with all singular values on the diagonal. If $\operatorname{rank}(A)=k$, then we have $k$ nonzero singular values: $\sigma_{1}, \sigma_{2}, \ldots, \sigma_{k}$, which are arranged as a diagonal matrix $\Sigma=\operatorname{diag}\left(\sigma_{1}, \sigma_{2}, \ldots, \sigma_{k}\right)$. Let $X \in C^{r \times k}$ be the first $k$ columns of $U$ and let $Y \in C^{s \times k}$ be the first $k$ columns of $V$. That is $U=\left[X U_{2}\right], \quad V=\left[Y V_{2}\right]$. Without loss of generality, we select $L=X Q$ and $R=Y Q$ for some nonzero $Q \in R^{k \times k}$. Using these, from Eq. (1) we have

$$
\tilde{A}=L^{T} A R=Q^{T}\left(X^{T} A Y\right) Q=Q^{T} \Sigma Q
$$

is a real symmetric matrix and this completes the proof.

Remark 3.1 The symmetric form of $A$ is not unique because $Q$ can be chosen in many ways. In particular, if we select a unit matrix as $Q$, then $\tilde{A}$ becomes a diagonal matrix.

Remark 3.2 Theorem 1 is, of course, applicable for real square matrices as a special case.

Example 3.1 The procedure outlined above can be illustrated by the following rectangular complex matrix from Example 4.1 in Adhikari [12]:

$$
A=\left[\begin{array}{ccc}
1.0+3.0 i & -2.0+1.0 i & 1.5-2.0 i \\
-2.0-3.0 i & 6.0+2.0 i & 7.0+2.0 i
\end{array}\right]
$$

Step 1: Performing singular value decomposition, we have 


$$
\begin{aligned}
U & =\left[\begin{array}{ccc}
0.0066+0.1956 i & -0.3975-0.8965 i \\
-0.5838-0.7879 i & -0.1669-0.1022 i
\end{array}\right] \\
S & =\left[\begin{array}{ccc}
10.4649 & 0 & 0 \\
0 & 4.2113 & 0
\end{array}\right] \\
V & =\left[\begin{array}{ccc}
0.3942 & -0.5809 & -0.7121 \\
-0.4679-0.3782 i & -0.3104+0.4537 i & -0.0058-0.5795 i \\
-0.5775-0.3862 i & -0.0418-0.5988 i & -0.2856+0.2747 i
\end{array}\right]
\end{aligned}
$$

Step 2: Observe that $\operatorname{rank}(A)=2$. We select matrix $X$ and $Y$ as follows

$$
\begin{aligned}
& X=U \\
& Y=\left[\begin{array}{cc}
0.3942 & -0.5809 \\
-0.4679-0.3782 i & -0.3104+0.4537 i \\
-0.5775-0.3862 i & -0.0418-0.5988 i
\end{array}\right]
\end{aligned}
$$

Step 3: Now arbitrarily select the matrix $Q$ as in [12]:

$$
Q=\left[\begin{array}{ll}
0.1021 & 0.2605 \\
1.4109 & 0.3519
\end{array}\right]
$$

so that

$$
\begin{aligned}
& L=X Q=\left[\begin{array}{cc}
-0.5602-1.2449 i & -0.1382-0.2645 i \\
-0.2951-0.2247 i & -0.2108-0.2412 i
\end{array}\right] \\
& R=Y Q=\left[\begin{array}{cc}
-0.7794 & -0.1018 \\
-0.4857+0.6016 i & -0.2311+0.0612 i \\
-0.1179-0.8843 i & -0.1652-0.3113 i
\end{array}\right]
\end{aligned}
$$

Using this $L$ and $R$ we obtain

$$
\tilde{A}=L^{T} A R=\left[\begin{array}{ll}
8.4923 & 2.3692 \\
2.3692 & 1.2317
\end{array}\right]
$$

which is a real nonsingular symmetric matrix.

Example 3.2 Consider a real asymmetric matrix from Example 4.2 in Adhikari [12]:

$$
A=\left[\begin{array}{ccc}
1.0 & -2.0 & 1.5 \\
12.0 & 6.0 & 7.0 \\
-2.0 & 4.0 & 9.0
\end{array}\right]
$$

Observe that $A$ has complex eigenvalues. Thus $A$ does not satisfy Taussky's condition of symmetrizability. In the following we will show that $A$ is a real symmetrizable matrix with the presented procedure. Note that $\operatorname{rank}(A)=3$. Selecting matrix $Q$ as in Adhikari [12]:

$$
Q=\left[\begin{array}{ccc}
0.0645 & -0.1647 & 0.1436 \\
-0.8923 & 0.2226 & 0.6155 \\
0.1611 & 0.2298 & 0.3708
\end{array}\right]
$$

and following the procedure described previously we obtain

$$
L=U Q=\left[\begin{array}{ccc}
0.1734 & 0.2198 & 0.3702 \\
0.3900 & -0.2469 & -0.1169 \\
-0.8026 & 0.1421 & 0.6215
\end{array}\right]
$$




$$
R=V Q=\left[\begin{array}{ccc}
0.7298 & -0.2298 & -0.2941 \\
-0.2638 & -0.2377 & -0.1620 \\
-0.4733 & 0.1420 & 0.6513
\end{array}\right]
$$

From these we have

$$
\tilde{A}=L^{T} A R=\left[\begin{array}{ccc}
7.0385 & -1.7960 & -4.4595 \\
-1.7960 & 1.0003 & 1.0316 \\
-4.4595 & 1.0316 & 3.9720
\end{array}\right]
$$

which is a real nonsingular symmetric matrix.

\section{Simultaneous symmetrization of two matrices}

The equation of motion of an $n$-degree-of-freedom linear undamped (nongyroscopic) system can be written as

$$
A \ddot{x}+B x=0
$$

where matrices $A$ and $B$ are of order $n \times n$; and where $x(t)$ is $n$-dimensional vectors of the generalized coordinates. For a classical system, it is assumed that $A$ is symmetric and positive definite, and that $B$ is symmetric and positive semi-definite. Here, however, no such restrictions are imposed on $A$ and $B$. Adhikari [12] has provided a sufficient condition under which the matrices $A$ and $B$ can be simultaneously transformed into symmetric matrices. That is, if $A^{T} B$ and $B A^{T}$ are symmetric, then $A$ and $B$ are simultaneously symmetrizable. Here we present an alternative result, which can be stated as in following:

Theorem 2: Let the Singular Value Decomposition of $A$ and $B$ be expressed as $A=U_{A} \Sigma_{A} V_{A}^{T}$ and $B=U_{B} \Sigma_{B} V_{B}^{T}$. Select $L=U_{A} Q$ and $R=V_{A} Q$ for some nonzero matrix $Q$. Then matrices $L^{T} A R$ and $L^{T} B R$ are symmetric if and only if

$$
\left(U_{A}^{T} U_{B}\right)^{T}\left(V_{A}^{T} V_{B}\right)=D
$$

is a diagonal matrix. In particular, if $Q$ is a unit matrix, then both $L^{T} A R$ and $L^{T} B R$ are diagonal.

Proof: It is easy to show that $A$ is symmetrizable by $L$ and $R$. In deed, we have

$$
\tilde{A}=L^{T} A R=Q^{T} U_{A}^{T}\left(U_{A} \Sigma_{A} V_{A}^{T}\right) V_{A} Q=Q^{T} \Sigma_{A} Q
$$

is a real symmetric. Next, we prove that $B$ is also symmetrizable by $L$ and $R$. In fact, we have

$$
\tilde{B}=L^{T} B R=Q^{T} U_{A}^{T}\left(U_{B} \Sigma_{B} V_{B}^{T}\right) V_{A} Q=Q^{T}\left(U_{A}^{T} U_{B}\right) \Sigma_{B}\left(V_{B}^{T} V_{A}\right) Q
$$

From Eq. (19), we have

$$
\left(U_{A}^{T} U_{B}\right)=\left(V_{B}^{T} V_{A}\right)^{T} D
$$

Substituting Eq. (23) into Eq. (22) we obtain

$$
\tilde{B}=Q^{T}\left(V_{B}^{T} V_{A}\right)^{T}\left(D \Sigma_{B}\right)\left(V_{B}^{T} V_{A}\right) Q
$$

Equation (24) shows that $\tilde{B}$ is a symmetric matrix if and only if $D \Sigma_{B}$ is diagonal. And this is true since both $D$ and $\Sigma_{B}$ are diagonal. The proof is complete.

Example 4.1. Consider an undamped system with matrix $A$ from Example 3.2 and

$$
B=\left[\begin{array}{ccc}
1.2044 & -5.4425 & 2.7013 \\
2.1007 & 0.8393 & 0.1894 \\
-1.8393 & 0.8953 & 2.5087
\end{array}\right]
$$

Numerical values of matrices $A$ and $B$ are taken from Example 5.2 of Adhikari [12]. Performing SVD we have 


$$
\begin{aligned}
& U_{A}=\left[\begin{array}{ccc}
0.0445 & -0.0108 & 0.9990 \\
0.9245 & -0.3784 & -0.0453 \\
0.3785 & 0.9256 & -0.0068
\end{array}\right] \\
& V_{A}=\left[\begin{array}{ccc}
0.6498 & -0.7383 & 0.1807 \\
0.4364 & 0.1676 & -0.8840 \\
0.6224 & 0.6533 & 0.4311
\end{array}\right] \\
& U_{B}=\left[\begin{array}{ccc}
0.9990 & -0.0108 & 0.0445 \\
-0.0453 & -0.3784 & 0.9245 \\
-0.0068 & 0.9256 & 0.3785
\end{array}\right] \\
& V_{B}=\left[\begin{array}{ccc}
0.1808 & -0.7383 & 0.6498 \\
-0.8840 & 0.1676 & 0.4364 \\
0.4311 & 0.6533 & 0.6224
\end{array}\right]
\end{aligned}
$$

It is easy to show that

$$
\left(U_{A}^{T} U_{B}\right)^{T}\left(V_{A}^{T} V_{B}\right)=\left[\begin{array}{ccc}
1.0 & 0 & 0 \\
0 & 1.0 & 0 \\
0 & 0 & 1.0
\end{array}\right]
$$

That is, the system matrices satisfy the condition of Theorem 2, thus the transforming matrices $L=U_{A} Q$ and $R=V_{A} Q$ symmetries $A$ and also symmetries $B$ as

$$
\tilde{B}=L^{T} B R=\left[\begin{array}{ccc}
2.8763 & -0.4671 & -1.4785 \\
-0.4671 & 0.5501 & 0.9468 \\
-1.4785 & 0.9468 & 2.1817
\end{array}\right]
$$

Example 4.2. Consider another undamped system with matrix $A$ same as in Example 3.2, and matrix $B$ from Example'5.3 in Adhikari [12]:

$$
B=\left[\begin{array}{ccc}
0.1955 & 1.8857 & -3.2199 \\
-2.1312 & -0.2236 & 0.3609 \\
1.0378 & 1.9501 & -1.5606
\end{array}\right]
$$

It is easy to check that matrices $A^{T} B$ and $B A^{T}$ are not symmetric. That is, $A$ and $B$ do not satisfy the sufficient condition of simultaneous symmetrization stated as Lemma 5.2 in Adhikari [12]. However, since

$$
\left(U_{A}^{T} U_{B}\right)^{T}\left(V_{A}^{T} V_{B}\right)=\left[\begin{array}{ccc}
-1.0 & 0 & 0 \\
0 & -1.0 & 0 \\
0 & 0 & 1.0
\end{array}\right]
$$

is a diagonal matrix, the system matrices satisfy the condition of Theorem 2, thus the transforming matrices $L$ and $R$ (computed in Example 3.2) also symmetries $B$ as

$$
\tilde{B}=L^{T} B R=\left[\begin{array}{ccc}
2.8763 & -0.4671 & -1.4785 \\
-0.4671 & 0.5501 & 0.9468 \\
-1.4785 & 0.9468 & 2.1817
\end{array}\right]
$$




\section{Simultaneous symmetrization of three matrices}

Consider a linear nonconservative system, whose equations of motion can be written as

$$
A \ddot{x}+C \dot{x}+B x=0
$$

where $A \in R^{N \times N}, B \in R^{N \times N}$ and $C \in R^{N \times N}$. Were these matrices symmetric and positive definite, they would, respectively, be the mass, stiffness, and viscous damping matrices. Here, however, no restrictions of symmetry or definiteness are imposed on these coefficient matrices. This brings an additional complication in simultaneous symmetrization. We will investigate what forms of restrictions should be imposed on the system matrices so that $A$, $B$ and $C$ can be simultaneously transformed into symmetric matrices. Our main result is the following:

Theorem 3: Let the Singular Value Decomposition of $A, B$ and $C$ be expressed as $A=U_{A} \Sigma_{A} V_{A}^{T}, B=U_{B} \Sigma_{B} V_{B}^{T}$, and $C=U_{C} \Sigma_{C} V_{C}^{T}$. Select $L=U_{A} Q$ and $R=V_{A} Q$ for some nonzero matrix $Q$. Then matrices $L^{T} A R, L^{T} B R$ and $L^{T} C R$ are symmetric if and only if $\left(U_{A}^{T} U_{B}\right)^{T}\left(V_{A}^{T} V_{B}\right)=D_{1}$ and $\left(U_{A}^{T} U_{C}\right)^{T}\left(V_{A}^{T} V_{C}\right)=D_{2}$ are diagonal.

This theorem can be easily proved in the same manner as in the proof of Theorem 2 above. The example considered below illustrates this result.

Example 5.1. Consider a damped dynamic system, whose equation of motion has the form Eq. (31), the coefficient matrices $A$ and $B$ are as in Example 4.1 and

$$
C=\left[\begin{array}{ccc}
0.1704 & 1.6376 & -1.3725 \\
1.6028 & 1.0300 & 1.9724 \\
0.9152 & 1.1257 & 0.1902
\end{array}\right]
$$

Note that the matrix $C$ does not satisfy Adhikari's condition of symmetrizability (see Lemma 6.5 in [12]). In fact, let $X$ and $Y$ be right and left eigenvectors, respectively, of the undamped system such that $B X=\Lambda A X$ and $Y^{T} B=\Lambda Y^{T} A$. It is easy to check that the matrix $Y^{T} C X$ is not symmetric. In other words, $C$ is not symmetrizable by Adhikari's method. In the following, we will show that the system under consideration is real symmetrizable by using the presented method. Indeed performing SVD for $C$, we obtain

$$
\begin{gathered}
U_{C}=\left[\begin{array}{ccc}
0.0070 & -0.9241 & 0.3821 \\
0.9124 & 0.1623 & 0.3758 \\
0.4093 & -0.3460 & -0.8443
\end{array}\right] \\
V_{C}=\left[\begin{array}{ccc}
0.6175 & -0.0923 & -0.7811 \\
0.4743 & -0.7485 & 0.4634 \\
0.6275 & 0.6566 & 0.4184
\end{array}\right]
\end{gathered}
$$

With the results of SVD obtained in Example 4.1, we have

$$
\left(U_{A}^{T} U_{C}\right)^{T}\left(V_{A}^{T} V_{C}\right)=\left[\begin{array}{ccc}
1.0 & 0 & 0 \\
0 & -1.0 & 0 \\
0 & 0 & -1.0
\end{array}\right]
$$

is diagonal. Thus the transforming matrices $L$ and $R$ (computed in Example 4.1) symmetries $A$ and $B$ as in Example 4.1, and also symmetries $C$ as

$$
\tilde{C}=L^{T} C R=\left[\begin{array}{ccc}
-0.1800 & 0.1239 & 0.3053 \\
0.1239 & -0.1188 & -0.4720 \\
0.3053 & -0.4720 & -0.7268
\end{array}\right]
$$




\section{Conclusions}

A method of symmetrization of asymmetric dynamical systems by means of equivalence transformation is introduced. This new method belongs to "symmetrization of the second kind" introduced by Adhikari [12], and the symmetrization method based on the similarity transformation is called "symmetrization of the first kind". Compared with existing methods, this new method of symmetrization not only keeps the advantages of symmetrization of the second kind, but also offers an easy numerical method to calculate symmetric forms of general matrices. Specifically, this method is much easier than calculating the symmetric form of the first kind using Taussky's factorization, and also avoids eigensolution calculation of the existing symmetrization of the second kind.

\section{References}

[1] K. Huseyin and H.H.E. Liepholz, Divergence Instability of Multiple Parameter Circulatory Systems, Q. Appl. Math. 31 (1973), $185-197$.

[2] I. Fawzy and R.E.D. Bishop, On the Nature of Resonance in Nonconservative Systems, J. Sound Vib. 55(4) (1977), 475-485.

[3] R.E.D. Bishop and W.G. Price, An Investigation Into the Linear Theory of Ship Response to Waves, J. Sound Vib. 62(3) (1979), $353-363$.

[4] W.R. Kliem, Symmetrizable Systems in Mechanics and Control Theory, ASME J. Appl. Mech. 59 (1992), 454-456.

[5] R.L. Huston and C.Q. Liu, Formulas for Dynamic Analysis, Marce Dekker, Inc., New York, N. Y.

[6] D.J. Inman, Dynamics of Asymmetric Nonconservative Systems, ASME J. Appl. Mech. 50 (1983), 199-203.

[7] M. Ahmadian and D.J. Inman, Classical Normal Modes in Asymmetric Nonconservative Dynamic Systems, AIAA $J$ 22(7) (1983), 1012-1015.

[8] T.K. Caughey and F. Ma, Complex Modes and Solvability of Nonconservative Linear Systems, ASME J. Appl. Mech. 60 (1993), 26-28.

[9] F. Ma and T.K. Caughey, Analysis of Linear Nonconservative Vibrations, ASME J. Appl. Mech. 62 (1995), 685-691.

[10] S.M. Shahruz and F. Ma, On Symmetrizability of Asymmetric Nonconservative Systems, ASME J. Appl. Mech. 56 (1989), 474-476.

[11] A. Cherng and M.K. Abdelhamid, On the Symmetrization of Asymmetric Finite Dimensional Linear Dynamic Systems, ASME J. Vib. Acoust. 115 (1993), 417-421.

[12] S. Adhikari, On Symmetrizable Systems of Second Kind, J. Appl. Mech. 67 (2000), 797-802.

[13] O. Taussky, Positive Definite matrices and Their Role in the Study of the Characteristic Roots of General Matrices, Pac. J. Math. 9 (1968), 893-896.

[14] S. Adhikari, Modal Analysis of Linear Asymmetric Nonconservative Systems, J. Eng. Mech. 125(12) (1999), $1372-1379$.

[15] K. Huseyin, Vibrations and Stability of Multiple - Parameter Systems, Sijthoff \& Noordhoff Inter. Publ., Netherlands, 1978.

[16] Kounadis and Smitses, Non-conservative systems with Symmetrizable Stiffness Matrices Exhibiting Limit Cycles, Int. J. Non-Linear mech. 32(3) (1997), 515-529. 

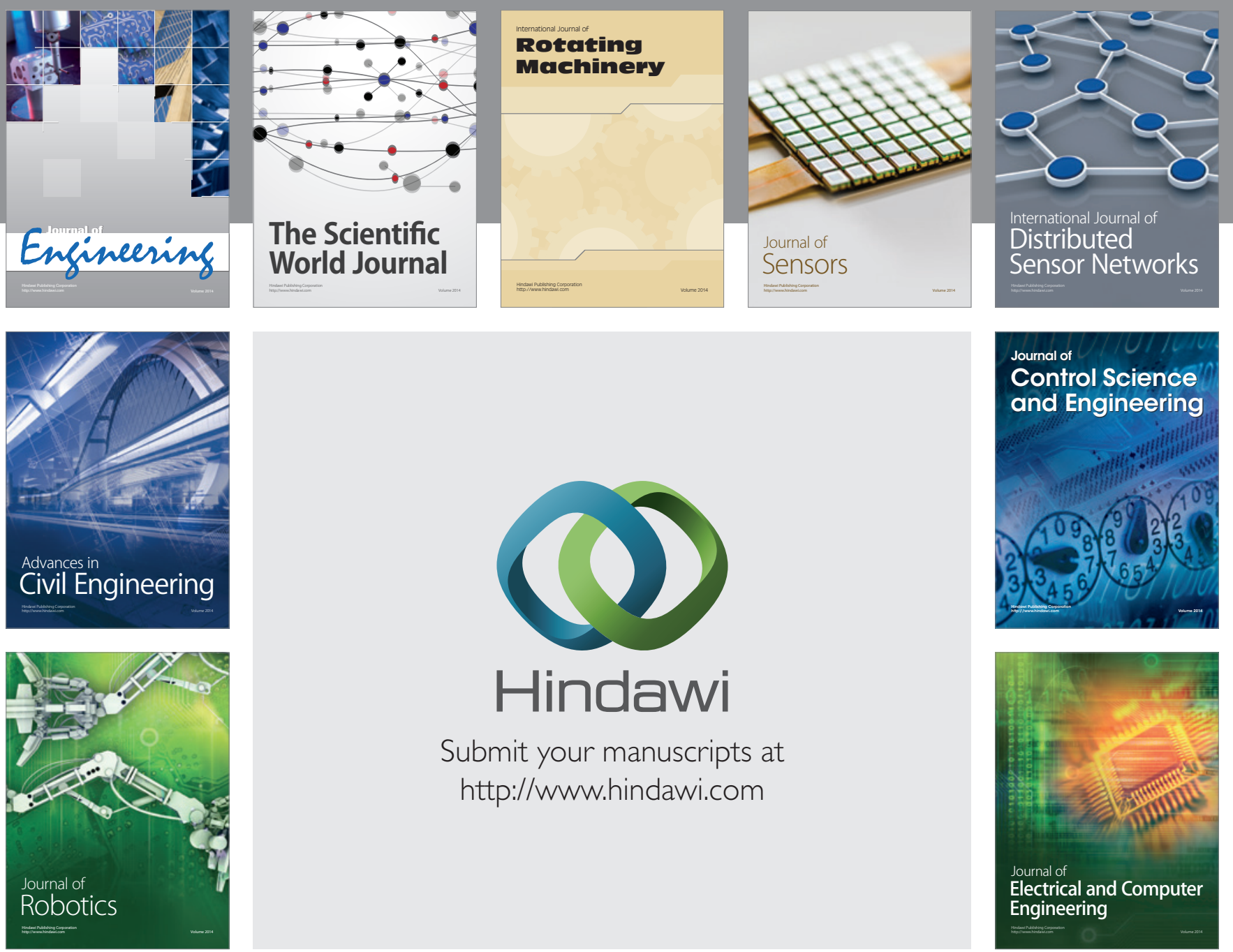

Submit your manuscripts at

http://www.hindawi.com
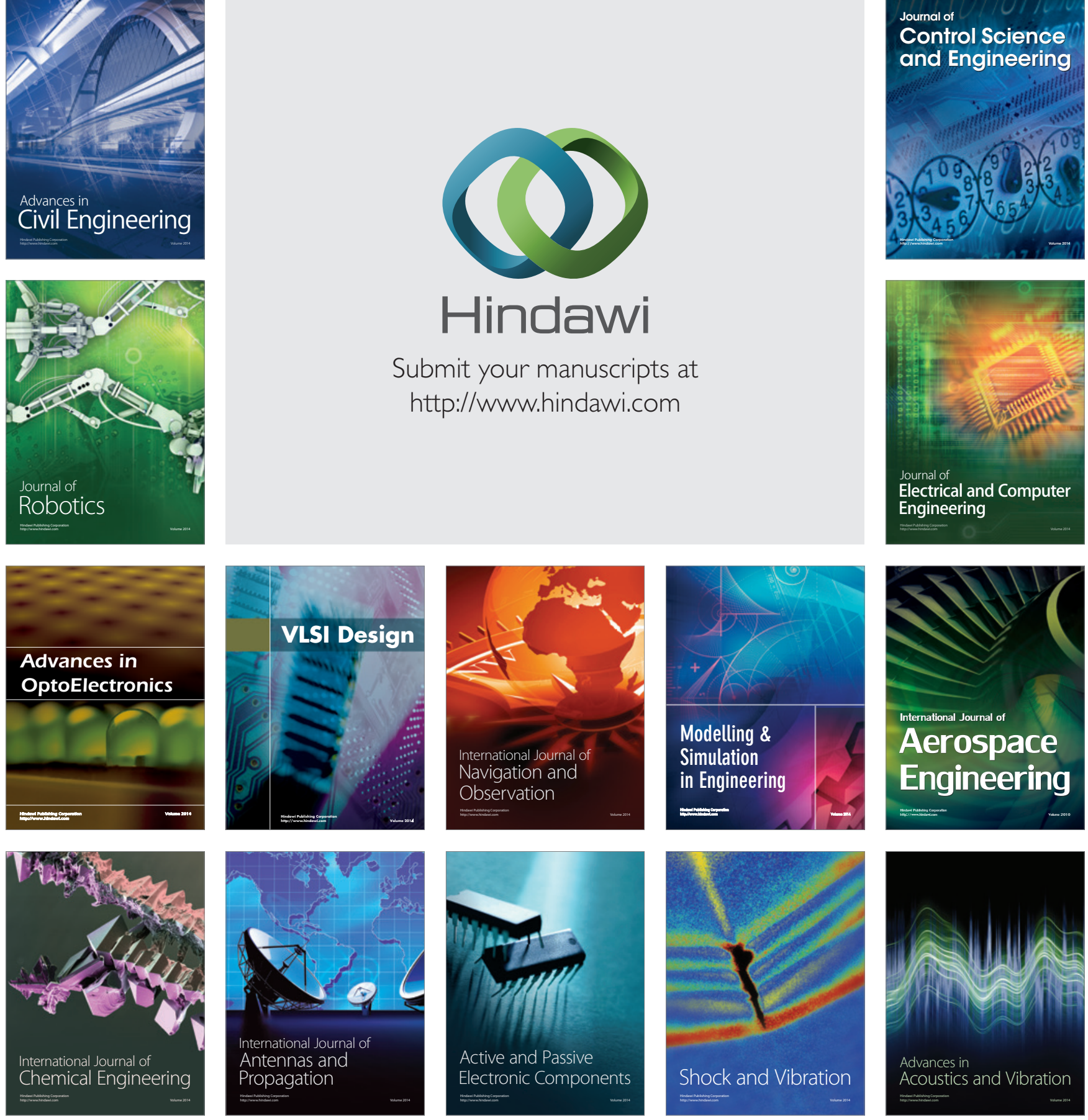